\title{
Editorial
}

\section{Inorganic Syntheses Assisted by Microwave Heating}

\section{Cristina Leonelli ${ }^{1, *}$ and Sridhar Komarneni ${ }^{2, *}$}

1 Department of Engineering "Enzo Ferrari”, University of Modena and Reggio Emilia, 41125 Modena, Italy

2 Materials Research Laboratory, Materials Research Institute, Pennsylvania State University, University Park, PA 16802, United States

* Authors to whom correspondence should be addressed;

E-Mails: cristina.leonelli@unimore.it (C.L.); sxk7@psu.edu (S.K.).

Academic Editor: Duncan H. Gregory

Received: 28 August 2015 / Accepted: 13 October 2015 / Published: 16 October 2015

This Special Issue on "Inorganic Syntheses Assisted by Microwave Heating" represents one of the few fully dedicated issues on inorganic microwave synthesis published by any international scientific journal and it features five papers and one review article.

Microwave irradiation has been used for many years to accelerate chemical reactions. The very first application of $2.45 \mathrm{GHz}$ microwaves in processing began with heating and cooking food in kitchens all over the world. However, the use of microwaves in analytical laboratories began later, starting with drying organic and inorganic samples in order to determine the amount of moisture [1] and later it was used for the dissolution or decomposition of not only biological but also geological materials [2-5].

Komarneni and Roy pioneered the use of microwaves in the liquid state for synthesis and published a seminal paper in Materials Letters in 1985 [6,7]. Unfortunately, the historical significance of this paper entitled "Titania gel spheres by a new sol-gel process" was not recognized by many as the title of this paper did not itself refer to microwaves. Nevertheless, the abstract clearly states that microwaves led to fast reaction as follows and we quote: "A new technique is devised for the preparation of solution derived titania gel spheres. The gel sphere preparation process involves the dispersal of the polymerized titanium ethoxide solution into a two-phase liquid suspension in kerosene followed by heating in a microwave oven. This is a simple and fast method for the preparation of xerogel spheres with diameter in the range of tens of micrometers which may be useful in coating technology by plasma-spraying".

Gedye et al., 1986 [8] and Gigure et al., 1986 [9] may have been the first to report on the use of microwaves for organic syntheses in 1986. These two papers are generally recognized as pioneering 
papers as they contained microwave ovens in their titles as follows: "The use of microwave ovens for rapid organic synthesis" and "Application of commercial microwave ovens to organic synthesis", respectively. Komarneni and Roy's paper predated these and might be considered the birth of "microwave assisted" reactions.

Later, in 1992, Komarneni coined the term "Microwave-hydrothermal (M-H) Process" [10] for reactions performed at temperatures above the boiling point of water or other solvents and pressures greater than $1 \mathrm{~atm}$. These two ground-breaking papers [6,10] revolutionized the field of microwave-assisted reactions in materials synthesis and processing in water and other liquids. Microwave-hydrothermal/solvothermal process has been widely developed by Komarneni's group for the synthesis of nano-sized oxide and metal materials and it has been shown to have many advantages such as (a) rapid heating to temperature of reaction, (b) increased reaction kinetics, (c) elimination of metastable phases and (d) forming of novel phases. The microwave-assisted reactions have spread world over resulting in extraordinary new scientific developments in the processing of oxide, semiconductor and metal nanophases as quantum dots, nanowires, nanorods, nanobelts, etc. Komarneni also coined the terms, "microwave-polyol process" and "microwave-solvothermal process" for synthesis of metals and other materials.

However, over the years a more complex view has emerged because of detailed work by many researchers, primarily in the field of organic chemistry. Inorganic chemists have exploited the advantages of microwave heating not only for its accelerated temperature ramp up, but also for shortening reaction times which could lead to metastable phases or high purity products.

The papers in this issue present different preparation procedures of solvothermal/hydrothermal synthesis as well as gas phase reactions using microwaves. Authors have reported nanopowder synthesis where a drastic reduction of treatment time, from hours to minutes, has been clearly shown. The effects of microwave irradiation on the different crystal morphologies and sizes and crystallinity have been thoroughly investigated and the results are presented. Organometallic syntheses are also reported with no significant differences compared to conventional solvothermal preparation. A discussion on microwave equipment and reactors with temperature and pressure monitoring issues in the microwave field is also reported.

This special issue also reports the recent knowledge in inorganic syntheses under microwave irradiation and presents the basic understanding of the heating mechanism and its effects on final yields and materials properties.

An efficient microwave-assisted synthesis of $\mathrm{TiO}_{2}$ : Using titanium tetraisopropoxide (TTIP), benzyl alcohol as the solvent, together with boric acid and oleic acid as the additive reagents, nanorods have been synthesized by the group coordianted by Ciccarella in Lecce, Italy [11]. The synthesized $\mathrm{TiO}_{2}$ anatase nanorods exhibited good photoactivity and completely degraded Rhodamine B solution within three hours.

Nanopowders of $\mathrm{Cu}, \mathrm{Mo}, \mathrm{W}, \mathrm{Mo}-\mathrm{Ni}$ and $\mathrm{Fe}-\mathrm{Co}$ were successfully prepared by using microwave plasma at a frequency of $2.45 \mathrm{GHz}$ by the group of Shih from Taiwan. [12].The processing conditions, such as plasma gas, carrier gas, cooling gas, precursor raw materials and feeding rate, were tuned to manipulate the particle size of the nanopowders efficiently.

Life-long work on microwave plasma gas-phase synthesis of simple and complex oxides and different type of ceramics, as different core/shell ceramic/organic shell nanoparticles, and Sn-based 
nanocomposites, has been reported in a detailed review by Szabò and Schlabach [13]. The fundamental basics, which are necessary to understand the mechanisms in particle synthesis and the influence of the relevant experimental parameters on the resulting particles and their properties have been addressed in this article. The benefit of using microwave plasma instead of conventional gas phase processes with respect to chemical reactivity and crystallite nucleation has been presented.

The group coordinated by Torres (Madrid, Spain) analyzed the use of microwave activation for the preparation of three new bromidotetracarboxylatodiruthenium(II,III) compounds of the type $\left[\mathrm{Ru}_{2} \mathrm{Br}\left(\mu-\mathrm{O}_{2} \mathrm{CC}_{6} \mathrm{H}_{4}-\mathrm{R}\right)_{4}\right]_{n}[\mathrm{R}=o-\mathrm{Me}(1), m-\mathrm{Me}(2), p$-Me (3)] [14]. The preparation by solvothermal and conventional methods was also reported as a comparison and these methods led to the same complexes in similar yields and with no appreciable differences in the properties of the compounds once the synthetic procedure was efficiently controlled. This paper cautions how closely the microwave assisted methods should be monitored before claiming a "microwave effect".

A group led by Łojkowski from Warsaw, Poland, report on the so-called "Microwave Solvothermal Synthesis (MSS)" technology for the preparation of nanoparticles of binary and complex oxides, $\mathrm{ZrO}_{2}$, $\mathrm{ZnO}$ and yttrium-aluminium garnet, $\mathrm{Y}_{3} \mathrm{Al}_{5} \mathrm{O}_{12}$ (YAG) [15]. The unique effect of the microwave heating allowed a very uniform nanosized powder preparation once the precise planning of a time-temperature region was scheduled to assure a high supersaturation of the reagents uniformly in the reactor vessel.

Morán's group from Madrid, Spain, reported on the preparation of functional oxides with complex crystalline structures [16]. This contribution explains how a broad variety of different microwave-assisted synthesis techniques opens up opportunities for the preparation of inorganic nanoparticles and nanostructures, especially in relation to solid-state reactivity exploiting single-mode microwaves using a TE $10 \mathrm{p}$ cavity, and microwave-assisted hydrothermal synthesis.

We hope that this Special Issue generates new interest not only in the pursuit of both inorganic and organic syntheses using microwaves but also in investigating the mechanisms of microwaveassisted reactions.

\section{References}

1. Hesek, J.A.; Wilson, R.C. Practical analysis of high-purity chemicals. X. Use of a microwave oven in in-process control. Anal. Chem. 1974, 46, 1160-1160.

2. Abu-Samra, A.; Steven Morris, J.; Koirtyohann, S.R. Wet ashing of some biological samples in a microwave oven. Anal. Chem. 1975, 47, 1475-1477.

3. Barrett, P.; Davidowski, L.J., Jr.; Penaro, K.W.; Copeland, T.R. Microwave oven-based wet digestion technique. Anal. Chem. 1978, 50, 1021-1023.

4. Nadkarni, R.A. Applications of microwave oven sample dissolution in analysis. Anal. Chem. 1984, 56, 2233-2237.

5. Smith, F.; Cousins, B.; Bozic, J.; Flora, W. The acid dissolution of sulfide mineral samples under pressure in a microwave oven. Anal. Chim. Acta 1985, 177, 243-245.

6. Komarneni, S.; Roy, R. Titania gel spheres by a new sol-gel process. Mat. Lett. 1985, 3, 165-167.

7. Komarneni, S. Microwave Memories. Chem. World 2009, 6, 38. 
8. Gedye, R.; Smith, F.; Westaway, K.; Ali, H.; Baldisera, L.; Laberge, L.; Rousell, J. The use of microwave ovens for rapid organic synthesis. Tetrahedron Lett. 1986, 27, 279-282.

9. Giguere, R.J.; Bray, T.L.; Duncan, S.M. Application of commercial microwave ovens to organic synthesis. Tetrahedron Lett. 1986, 27, 4945-4948.

10. Komarneni, S.; Roy, R.; Li, Q.H. Microwave-hydrothermal synthesis of ceramic powders. Mater. Res. Bull. 1992, 27, 1393-1405.

11. Carlucci, C.; Scremin, B.F.; Sibillano, T.; Giannini, C.; Filippo, E.; Perulli, P.; Capodilupo, A.L.; Corrente, G.A.; Ciccarella, G. Microwave-assisted synthesis of boron-modified $\mathrm{TiO}_{2}$ nanocrystals. Inorganics 2014, 2, 264-277.

12. Chau, J.L.H.; Yang, C.-C.; Shih, H.-H. Microwave plasma production of metal nanopowders. Inorganics 2014, 2, 278-290.

13. Szabó, D.V.; Schlabach, S. Microwave plasma synthesis of materials-From physics and chemistry to nanoparticles: A materials scientist's viewpoint. Inorganics 2014, 2, 468-507.

14. Delgado-Martínez, P.; Elvira-Bravo, A.; González-Prieto, R.; Priego, J.L.; Jimenez-Aparicio, R.; Torres, M.R. Synthesis of $\mathrm{Ru}_{2} \mathrm{Br}\left(\mu-\mathrm{O}_{2} \mathrm{CC}_{6} \mathrm{H}_{4}-\mathrm{R}\right)_{4}(\mathrm{R}=o$-Me, $m$-Me, $p$-Me) using microwave activation: Structural and magnetic properties. Inorganics 2014, 2, 524-536.

15. Lojkowski, W.; Leonelli, C.; Chudoba, T.; Wojnarowicz, J.; Majcher, A.; Mazurkiewicz, A. High-energy-low-temperature technologies for the synthesis of nanoparticles: Microwaves and high pressure. Inorganics 2014, 2, 606-619.

16. Prado-Gonjal, J.; Schmidt, R.; Morán, E. Microwave-assisted routes for the synthesis of complex functional oxides. Inorganics 2015, 3, 101-117.

(C) 2015 by the authors; licensee MDPI, Basel, Switzerland. This article is an open access article distributed under the terms and conditions of the Creative Commons Attribution license (http://creativecommons.org/licenses/by/4.0/). 